\title{
Novel Bis-(3-Cyano-2-Pyridones) Derivatives: Synthesis and Fluorescent Properties
}

Nawel Mehiaoui ${ }^{\mathrm{a}}$, Zahira Kibou ${ }^{\mathrm{a}, \mathrm{b}}$, Thibault Gallavardin ${ }^{\mathrm{c}}$, Stéphane Leleu ${ }^{\mathrm{C}}$, Xavier Franck ${ }^{\mathrm{C}}$, Ricardo F. Mendes ${ }^{d}$, Filipe A. Almeida Paz ${ }^{\mathrm{d}}$, Artur M. S. Silva ${ }^{\mathrm{e}}$, Noureddine ChoukchouBraham $^{\mathrm{a}}$

${ }^{a}$ Laboratoire de Catalyse et Synthèse en Chimie Organique, Faculté des Sciences, Université de Tlemcen, BP 119, 13000 Tlemcen, Algeria

b Centre Universitaire Ain Témouchent, Institut des Sciences, BP 284, 46000 Ain Témouchent, Algeria

${ }^{c}$ COBRA (UMR6014 and FR 3038) Normandie Univ, CNRS, INSA Rouen, UNIROUEN 76000 Rouen, France

${ }^{\mathrm{d}}$ Department of Chemistry, CICECO-Aveiro Institute of Materials, University of Aveiro, 3810-193 Aveiro, Portugal

${ }^{\mathrm{e}}$ Department of Chemistry, QOPNA \& LAQV-REQUIMTE, University of Aveiro, 3810-193 Aveiro, Portugal

\section{*Corresponding author: zahira_kibou@yahoo.fr}

\section{Abstract}

Novel substituted bis-(3-cyano-2-pyridone) derivatives were prepared via a powerful method using enaminonitriles push-pull dienes as key building blocks. The synthesis was performed in three-steps from easily accessible starting materials in good yields. All target products were structurally elucidated by spectroscopic data. The reaction mechanism for formation of bis (3cyano-2-pyridone) derivatives was proposed. The structures of enaminonitriles 3a and bispyridones $\mathbf{4 c}$ were confirmed by single-crystal X-ray diffraction. The florescent study of some bis (3-cyano-2-pyridones) derivatives was reported.

\section{Graphical abstract}

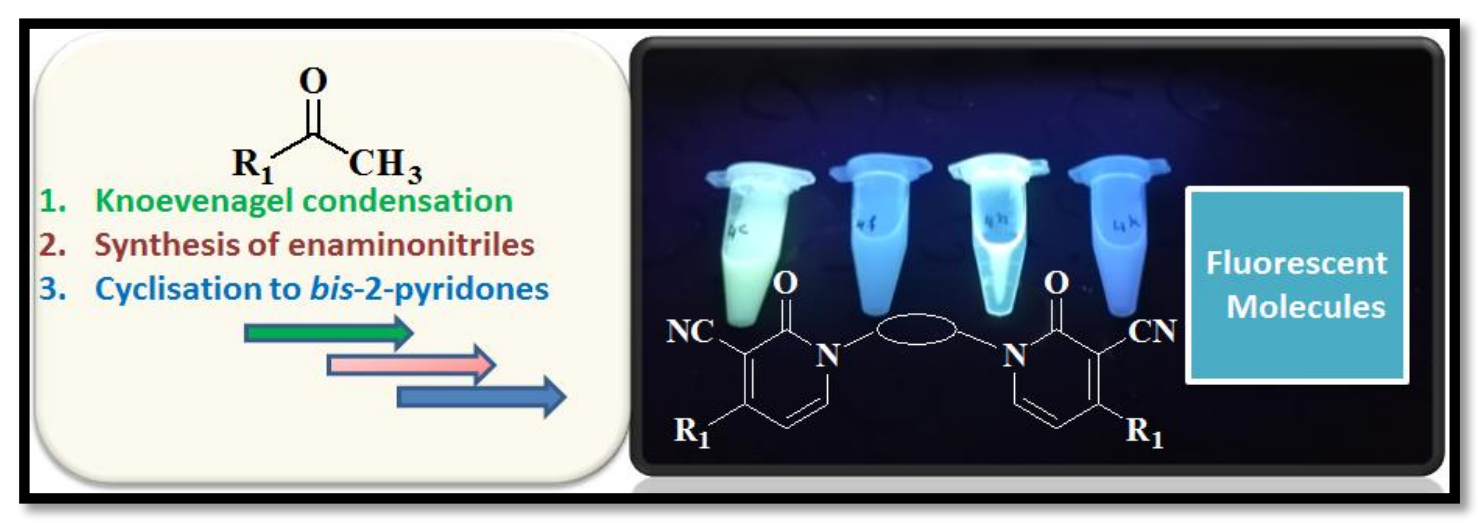




\section{Keywords}

Bis (2-pyridones) · Diamines $•$ Enaminonitriles $•$ Green synthesis $•$ molecular fluorescence

\section{Introduction}

The development of new and efficient protocols to prepare nitrogen-heterocyclic compounds has received great interest in organic chemistry [1-2]. Numerous synthetic methods to prepare $N$-heterocyclic compounds have been reported over the years [3-4] since these structures are present in many natural compounds, as for example 2-pyridones (Figure 1).

2-Pyridone derivatives constitute an important class of heterocycles due to their diverse biological properties, such as antibacterial and antifungal activities. [5-6].They are also described as inhibitors of DNA gyrase, [7] partial agonist of nicotinic cholinergic receptors (nAChRs), [8]. Phosphodiesterase 3 (PDE 3) inhibitors (milrinone, amrinone), [9-11] tissue factor VIIa inhibitor, [12] selective AMPA receptor antagonist [13-15]. 2-Pyridones scaffolds finding versatile applications in pharmaceuticals [16], agrochemicals [17], and organic functional materials [7].

Moreover, 2-pyridone ring is one of the most classes of fluophores, with several photo functional applications [18], this skeleton exhibit a strong emission in different regions of spectrum. Recently, they are a much interest in the development of convenient methodologies of the synthesis of 2-pyridones from simple and available starting materials to their fluorescent properties [19-21].

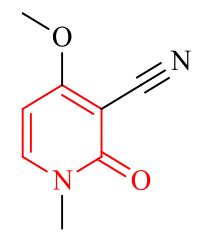

Ricinine

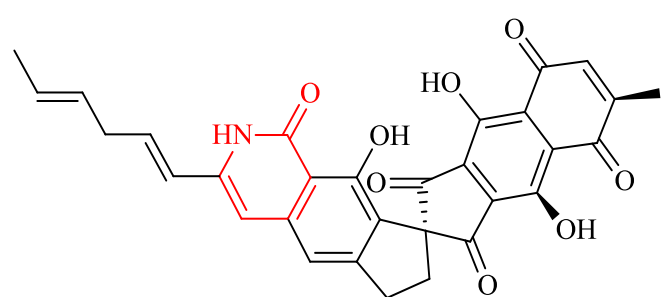

Fredericamycine A

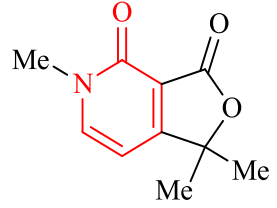

Cerpegine

Figure 1. Some natural products bearing a pyridone moiety

According to the literature, 2-pyridone derivatives are one of the most popular scaffolds used in the synthesis of heterocycles such quinolines, $\beta$-lactams, quinolizidines, pyridines, piperidines, and indolizidines and many alkaloids systems and peptides [22-23]. In recent studies some of these compounds proved to have a remarkable effect in vitro as powerful cell growth inhibitors for many types of human tumour [24]. However, there are only a few methods reported in literature for their preparation. These include multicomponent reactions 
(MCRs) using Meldrum's acid with substituted benzaldehyde and the active methylene compounds [25]. Another method was reported by Cocco et al. from a sequence of reactions using 3-amino-3-dialkylaminopropenoates with bis-(2,4,6-trichlorophenyl)malonate [26-27]. Demuner et al. have also reported the synthesis of bis-2-pyridones from commercial dehydroacetic acid (DHA). DHA was converted into 4-hydroxy-6-methylpyridin-2(1H)-ones, which was then condensed with several aliphatic aldehydes to produce bis-2-pyridones[28]. Recently, Sanad et al. have employed bis-(2-cyanoacetamide) and benzylidenemalononitrile in the presence of a variety of base catalysts, including DBU, piperidine, DABCO, and triethylamine to prepare bis-(6-amino-2-oxo-4-phenyl-1,2-dihydropyridine-3,5-dicarbonitrile) derivatives [29].

Following to our interest on the synthesis of heterocyclic compounds [30-35], we describe here a synthesis of a new class of bis-2-pyridone compounds (Figure 2) with fluorescent properties, there is no publication about fluorescent properties of bis-pyridones.<smiles>[R]c1c([Y])c([R])n([R])c(=O)c1[R]</smiles><smiles>[R]c1ccn(C2CCCC2)c(=O)c1C#N</smiles>

Figure 2. Structures of 3-cyano-2-pyridones and substituted bis-2-pyridones

\section{Experimental}

\section{General information}

The melting points were measured using a Bank Kofler Heizbank apparatus standard WME $50-260^{\circ} \mathrm{C}$ without particular correction. IR spectra were performed on solid samples using a Fourier transform Perkin Elmer Spectrum with ATR accessory. Only significant absorptions are listed. The ${ }^{1} \mathrm{H}$ and ${ }^{13} \mathrm{C}$ NMR spectra were recorded on a Bruker AC 400 spectrometers at 400 and $100 \mathrm{MHz}$, respectively. Samples were recorded in $\mathrm{CDCl}_{3}$ solutions using TMS as an internal standard. The chemical shifts are expressed in $\delta$ units (ppm) and quoted downfield from TMS. The multiplicities are reported as: s, singlet; $d$, doublet; $t$, triplet; q, quartet; $m$, multiplet. A series of COSY, NOESY, HSQC and HMBC NMR experiments were carried out to assign both ${ }^{1} \mathrm{H}$ and ${ }^{13} \mathrm{C}$ signals. UV-visible absorption spectra were measured with an Agilent Cary 60. Fluorescence was measured with a corrected Horiba Joblin Yvon Fluorolog 3 with diluted samples $(\mathrm{OD}<0.1)$. Fluorescence quantum Yields were measured using quinine 
sulfate in $\mathrm{H}_{2} \mathrm{SO}_{4}$ as a standard $(\mathrm{QY}=0.53)$ with excitation at $350 \mathrm{~nm}$. Single crystals of 3a and $\mathbf{4 c}$ were manually harvested from the crystallization vials and immersed in highly viscous FOMBLIN Y perfluoropolyether vacuum oil (LVAC 140/13, Sigma-Aldrich) to avoid degradation caused by the evaporation of the solvent [36]. Crystals were mounted Mi Te Gen Micro Loops with the help of a Stemi 2000 stereomicroscope equipped with Carl Zeiss lenses. Crystal data was collected at 150(2) K on a Bruker X8 Kappa APEX II CCD area-detector diffractometer (Mo K $\alpha$ graphite-monochromated radiation, $\lambda=0.71073 \AA$ ) controlled by the APEX3 software package [37] and equipped with an Oxford Cryosystems Series 700 cryostream monitored remotely using the software interface Cryopad [38]. Diffraction images were processed using the software package SAINT ${ }^{+}$, [39] and data were corrected for absorption by the multiscan semi-empirical method implemented in SADABS 2016/2 [40].

Structures were solved using the algorithm implemented in SHELXT-2014/5, [41] which allowed the immediate location of almost all of the heaviest atoms composing the molecular unit. The remaining missing and misplaced non-hydrogen atoms were located from difference Fourier maps calculated from successive full-matrix least-squares refinement cycles on F2 using the latest SHELXL from the 2018/3 release [42]. All structural refinements were performed using the graphical interface ShelXle [43].

Hydrogen atoms bound to carbon were placed at their idealized positions using appropriate HFIX instructions in SHELXL: 43 (aromatic carbon atoms), 23 (for the $-\mathrm{CH}_{2}-$ groups), 127 (for the disordered $-\mathrm{CH}_{3}$ group) and 1 (for the disordered $-\mathrm{CH}_{2}-$ groups). These hydrogen atoms were included in subsequent refinement cycles with isotropic thermal displacements parameters $\left(U_{\text {iso }}\right)$ fixed at $1.2 \times U_{\text {eq }}$ of the parent carbon atoms. We note that the type of hydrogen treatment for the latter disordered $-\mathrm{CH}_{2}-$ moieties was indeed necessary due to the fact that the molecule sits, alongside with the described disorder, over an inversion centre which limited the usage of the riding motion approximation for these atoms.

The last difference Fourier map synthesis showed for 3a, the highest peak $\left(0.302 \mathrm{e}^{-3}\right)$ and the deepest hole $\left(-0.243 \mathrm{e}^{-3}\right)$ located at 0.25 and $0.17 \AA$ from $\mathrm{H} 10 \mathrm{~A}$ and $\mathrm{H} 10 \mathrm{E}$, respectively; and for $\mathbf{4 c}$, the highest peak $\left(0.166 \mathrm{e}^{-3}\right)$ and the deepest hole $\left(-0.179 \mathrm{e}^{-3}\right)$ located at 0.90 and $0.86 \AA$ from C7 and N1, respectively. Structural drawings have been created using the software package Crystal Impact Diamond [44].

Crystallographic data (including structure factors) for the crystal structures of compound $\mathbf{4 c}$ have been deposited with the Cambridge Crystallographic Data Centre. Copies of the data can be obtained free of charge on application to CCDC, 12 Union Road, Cambridge CB2 2EZ, U.K. FAX: (+44) 1223 336033. E-mail: deposit@ccdc.cam.ac.uk. 


\section{Synthesis}

\section{General procedure for the preparation of compounds 2a-d:}

A mixture of methyl ketones 1a-d $(10 \mathrm{mmol})$, ethyl 2-cyanoacetate $(10 \mathrm{mmol})$, and ammonium acetate $(10 \mathrm{mmol})$ was heated at $50^{\circ} \mathrm{C}$ for $3 \mathrm{~h}$ in the presence of acetic acid $(0.06$ $\mathrm{mL}$ ). The progress of the reaction was monitored by TLC. After completion of the reaction, the reaction mixture was diluted with water $(5 \mathrm{~mL})$ and was extracted with dichloromethane (3 x $10 \mathrm{~mL}$ ). The organic extracts were combined and further washed with water and dried over $\mathrm{MgSO}_{4}$. Finally, the solvent was removed under vacuum and the resulting product was purified by distillation to give pure compounds $\mathbf{2 a - d}$.

\section{Ethyl 2-cyano-3-phenylbut-2-enoate 2a}

Using acetophenone $(1.20 \mathrm{~g}, 10 \mathrm{mmol})$, ethyl cyanoacetate $(1.13 \mathrm{~g}, 10 \mathrm{mmol})$ and ammonium acetate $(0.77 \mathrm{~g}, 10 \mathrm{mmol})$ it was obtained2a. Yield: $1.01 \mathrm{mg}(47 \%)$, as brown oil. IR $\left(v_{\max } / \mathrm{cm}^{-}\right.$ $\left.{ }^{1}\right): 1592(\mathrm{C}=\mathrm{C}), 1650(\mathrm{C}=\mathrm{O}), 2223(\mathrm{CN}) .{ }^{1} \mathrm{HNMR}\left(400 \mathrm{MHz}, \mathrm{CDCl}_{3}\right): \delta_{\mathrm{H}} 1.38(3 \mathrm{H}, \mathrm{t}, J=7.2$ $\left.\mathrm{Hz}, \mathrm{CO}_{2} \mathrm{CH}_{2} \mathrm{CH}_{3}\right), 2.69\left(3 \mathrm{H}, \mathrm{s}, \mathrm{C}=\mathrm{C}-\mathrm{CH}_{3}\right), 4.34\left(2 \mathrm{H}, \mathrm{q}, J=7.2 \mathrm{~Hz}, \mathrm{CH}_{2}\right), 7.15-7.40(5 \mathrm{H}, \mathrm{m}$, Ar). ${ }^{13} \mathrm{C} \mathrm{NMR}\left(101 \mathrm{MHz}, \mathrm{CDCl}_{3}\right): \delta_{\mathrm{C}} 14.2\left(\mathrm{CH}_{3}\right), 23.2\left(\mathrm{OCH}_{2}-\mathrm{CH}_{3}\right), 62.0\left(\mathrm{OCH}_{2}-\mathrm{CH}_{3}\right), 105.0$ $[\mathrm{C}=\mathrm{C}(\mathrm{CN})], 115.8(\mathrm{CN}), 127.3\left(\mathrm{C}_{\text {arom }}\right), 128.7\left(\mathrm{C}_{\text {arom }}\right), 130.4\left(\mathrm{C}_{\text {arom }}\right), 140.3\left(\mathrm{C}_{\text {arom }}\right), 162.0$ $(\mathrm{C}=\mathrm{O}), 172.1[\mathrm{C}=\mathrm{C}(\mathrm{CN})]$.

\section{Ethyl 2-cyano-3-p-tolylbut-2-enoate 2b}

Using 1-p-tolyl-ethanone (1.34 g, $10 \mathrm{mmol})$, ethyl cyanoacetate $(1.13 \mathrm{~g}, 10 \mathrm{mmol})$ and ammonium acetate $(0.77 \mathrm{~g}, 10 \mathrm{mmol})$ it was obtained2b. Yield: $1.03 \mathrm{mg}(45 \%)$, as brown oil.IR $\left(v_{\max } / \mathrm{cm}^{-1}\right)$ : $1680(\mathrm{C}=\mathrm{C}), 1727(\mathrm{C}=\mathrm{O}), 2223(\mathrm{CN}) .{ }^{1} \mathrm{H}$ NMR $\left(400 \mathrm{MHz}, \mathrm{CDCl}_{3}\right): \delta_{\mathrm{H}} 1.38$ $\left(3 \mathrm{H}, \mathrm{t}, J=7.1 \mathrm{~Hz}, \mathrm{CO}_{2} \mathrm{CH}_{2} \mathrm{CH}_{3}\right), 2.39\left(3 \mathrm{H}, \mathrm{s}, \mathrm{Ph}-\mathrm{CH}_{3}\right), 2.67\left(3 \mathrm{H}, \mathrm{s}, \mathrm{C}=\mathrm{C}-\mathrm{CH}_{3}\right), 4.33(2 \mathrm{H}, \mathrm{q}, J$ $\left.=7.1 \mathrm{~Hz}, \mathrm{CH}_{2}\right), 7.38-7.06(\mathrm{~m}, 4 \mathrm{H}, \mathrm{Ar}) .{ }^{13} \mathrm{C} \mathrm{NMR}\left(101 \mathrm{MHz}, \mathrm{CDCl}_{3}\right): \delta_{\mathrm{C}} 14.0\left(\mathrm{CH}_{3}\right), 23.1$ $\left(\mathrm{Ph}-\mathrm{CH}_{3}\right), 26.7\left(\mathrm{OCH}_{2}-\mathrm{CH}_{3}\right), 61.8\left(\mathrm{OCH}_{2} \mathrm{CH}_{3}\right), 104.4[\mathrm{C}=\mathrm{C}(\mathrm{CN})], 116.4(\mathrm{CN}), 127.3\left(\mathrm{C}_{\text {arom }}\right)$, $129.4\left(\mathrm{C}_{\text {arom }}\right), 137.4\left(\mathrm{C}_{\text {arom }}\right), 141.0\left(\mathrm{C}_{\text {arom }}\right) ; 162.3(\mathrm{C}=\mathrm{O}), 172.3[\mathrm{C}=\mathrm{C}(\mathrm{CN})]$.

\section{Ethyl 2-cyano-3-(2,4-dichlorophenyl)but-2-enoate 2c}

Using 1-(2, 4-dichlorophenyl)ethanone, (1.89 g, $10 \mathrm{mmol})$ of ethyl cyanoacetate $(1.13 \mathrm{~g}, 10$ $\mathrm{mmol})$ and ammonium acetate $(0.77 \mathrm{~g}, 10 \mathrm{mmol})$ it was obtained 2c. Yield: $1.42 \mathrm{mg}(50 \%)$, as brown oil.IR $(\mathrm{KBr})\left(v_{\max } / \mathrm{cm}^{-1}\right)$ : $1614(\mathrm{C}=\mathrm{C}), 1737(\mathrm{C}=\mathrm{O}), 2230(\mathrm{CN}) .{ }^{1} \mathrm{H}$ NMR $(400 \mathrm{MHz}$, $\left.\mathrm{CDCl}_{3}\right): \delta_{\mathrm{H}} 1.18\left(3 \mathrm{H}, \mathrm{t}, J=7.1 \mathrm{~Hz}, \mathrm{CO}_{2} \mathrm{CH}_{2} \mathrm{CH}_{3}\right), 2.50\left(3 \mathrm{H}, \mathrm{s}, \mathrm{C}=\mathrm{C}-\mathrm{CH}_{3}\right), 4.12(2 \mathrm{H}, \mathrm{q}, J=7.1$ $\left.\mathrm{Hz}, \mathrm{CH}_{2}\right), 7.45(\mathrm{~s}, 1 \mathrm{H}, \mathrm{Ar}), 7.13(\mathrm{~d}, J=5.7 \mathrm{~Hz}, 1 \mathrm{H}, \mathrm{Ar}), 6.98(\mathrm{~d}, J=8.3 \mathrm{~Hz}, 1 \mathrm{H}) .{ }^{13} \mathrm{C} \mathrm{NMR}$ $\left(101 \mathrm{MHz}, \mathrm{CDCl}_{3}\right): \delta_{\mathrm{C}} 13.5\left(\mathrm{CH}_{3}\right), 26.2\left(\mathrm{OCH}_{2}-\mathrm{CH}_{3}\right), 62.1\left(\mathrm{OCH}_{2}-\mathrm{CH}_{3}\right), 109.0[\mathrm{C}=\mathrm{C}(\mathrm{CN})]$, 114.6(CN), $127.3\left(\mathrm{C}_{\text {arom }}\right), 128.8\left(\mathrm{C}_{\text {arom }}\right), 135.1\left(\mathrm{C}_{\text {arom }}\right), 136.8\left(\mathrm{C}_{\text {arom }}\right), 159.9(\mathrm{C}=\mathrm{O}), 166.8$ $[\mathrm{C}=\mathrm{C}(\mathrm{CN})]$.

\section{Ethyl 2-cyano-3-(4-nitrophenyl)but-2-enoate 2d}

Using of 1-(4-nitrophenyl) ethanone (1.65 g, $10 \mathrm{mmol})$, ethyl cyanoacetate and ammonium acetate $(1.13 \mathrm{~g}, 10 \mathrm{mmol})$ it was obtained 2d. Yield: $(62 \%)$, as white solid, $\mathrm{mp} 154^{\circ} \mathrm{C}$.IR $\left(v_{\max } / \mathrm{cm}^{-1}\right): 1614(\mathrm{C}=\mathrm{C}), 1727(\mathrm{C}=\mathrm{O}), 2223(\mathrm{CN}) .{ }^{1} \mathrm{HNMR}\left(400 \mathrm{MHz}, \mathrm{CDCl}_{3}\right): \delta_{\mathrm{H}} 1.57(3 \mathrm{H}, \mathrm{t}$, $\left.J 7.1 \mathrm{~Hz}, \mathrm{CO}_{2} \mathrm{CH}_{2} \mathrm{CH}_{3}\right), 2.41\left(3 \mathrm{H}, \mathrm{s}, \mathrm{Ph}-\mathrm{CH}_{3}\right), 3.57\left(2 \mathrm{H}, \mathrm{q}, J=7.1 \mathrm{~Hz}, \mathrm{CH}_{2}\right), 5.33(3 \mathrm{H}, \mathrm{s}$, $\left.\mathrm{C}=\mathrm{C}-\mathrm{CH}_{3}\right), 7.15-7.95(\mathrm{~m}, 4 \mathrm{H}, \mathrm{Ar}),{ }^{13} \mathrm{C} \mathrm{NMR}\left(101 \mathrm{MHz}, \mathrm{CDCl}_{3}\right): \delta_{\mathrm{C}} 21.3\left(\mathrm{CH}_{3}\right), 88.0\left(\mathrm{OCH}_{2^{-}}\right.$ 
$\left.\mathrm{CH}_{3}\right), 111.0\left(\mathrm{OCH}_{2}-\mathrm{CH}_{3}\right), 117.2[\mathrm{C}=\mathrm{C}(\mathrm{CN})], 124.2\left(\mathrm{C}_{\text {arom }}\right), 124.4\left(\mathrm{C}_{\text {arom }}\right), 127.1\left(\mathrm{C}_{\text {arom }}\right), 129.3$ $\left(\mathrm{C}_{\text {arom }}\right), 136.2(\mathrm{CN}), 159.8(\mathrm{C}=\mathrm{O}), 160.2[\mathrm{C}=\mathrm{C}(\mathrm{CN})]$.

\section{General procedure for the preparation of compounds 3a-d}

An equimolar mixture of compound 2a-d (10 mmol) and of DMFDMA (10 mmol) was stirred at room temperature. When the reaction was completed (monitored by TLC), the solid obtained was washed with absolute ethanol and diethyl ether $\left(\mathrm{Et}_{2} \mathrm{O}\right)$ to provide pure products (3a-d).

\section{Ethyl 2-cyano-5-dimethylamino-3-phenylpenta-2, 4-dienoate 3a}

Using $2 \mathbf{a}(2.15 \mathrm{~g}, 10 \mathrm{mmol})$ and DMFDMA $(1.19 \mathrm{~g}, 10 \mathrm{mmol})$ it was obtained compound 3a $(69 \%)$, as yellow solid, $\mathrm{mp} 130^{\circ} \mathrm{C}$. IR $\left(v_{\max } / \mathrm{cm}^{-1}\right): 1508(\mathrm{C}=\mathrm{C}), 1613(\mathrm{C}=\mathrm{C}), 1677(\mathrm{C}=\mathrm{O})$, $2192(\mathrm{CN}) .{ }^{1} \mathrm{H}$ NMR $\left(400 \mathrm{MHz}, \mathrm{CDCl}_{3}\right): \delta_{\mathrm{H}} 1.22\left(3 \mathrm{H}, \mathrm{t}, J=7.1 \mathrm{~Hz}, \mathrm{CH}_{2}-\mathrm{CH}_{3}\right), 2.93(3 \mathrm{H}, \mathrm{s}$, $\left.\mathrm{NCH}_{3}\right), 3.01\left(3 \mathrm{H}, \mathrm{s}, \mathrm{NCH}_{3}\right), 4.14\left(2 \mathrm{H}, \mathrm{q}, J=7.1 \mathrm{~Hz}, \mathrm{CH}_{2}-\mathrm{CH}_{3}\right), 6.62(1 \mathrm{H}, \mathrm{d}, J=12.6 \mathrm{~Hz}$, $\mathrm{CH}=\mathrm{CH}-\mathrm{N}), 7.12(1 \mathrm{H}, \mathrm{d}, J=12.6 \mathrm{~Hz}, \mathrm{CH}=\mathrm{CH}-\mathrm{N}), 7.21-7.45(5 \mathrm{H}, \mathrm{m}, \mathrm{Ar}) .{ }^{13} \mathrm{C} \mathrm{NMR}(101$ $\left.\mathrm{MHz}, \mathrm{CDCl}_{3}\right)$ : $\delta_{\mathrm{C}} 14.9\left(\mathrm{CH}_{2}-\mathrm{CH}_{3}\right), 38.0\left(\mathrm{NCH}_{3}\right), 45.9\left(\mathrm{NCH}_{3}\right), 59.9\left(\mathrm{CH}_{2}-\mathrm{CH}_{3}\right), 82.9$ $[\mathrm{C}=\mathrm{C}(\mathrm{CN})], 99.6(\mathrm{C}=\mathrm{C}-\mathrm{N}), 120.3(\mathrm{CN}), 128.7-138.3\left(6 \mathrm{C}_{\text {arom }}\right), 158.0(\mathrm{C}=\mathrm{C}-\mathrm{N}), 165.3(\mathrm{C}=\mathrm{O})$, 169.0 [C $=\mathrm{C}(\mathrm{CN})]$. Calcd. for $\mathrm{C}_{16} \mathrm{H}_{18} \mathrm{~N}_{2} \mathrm{O}_{2}: 71.09 \mathrm{C}, 6.71 \mathrm{H}, 10.36 \mathrm{~N}$. Found: $70.28 \mathrm{C}, 6.67 \mathrm{H}$ and $10.71 \mathrm{~N}$.

\section{Ethyl 2-Cyano-5-dimethylamino-3-p-tolylpenta-2, 4-dienoate 3b}

From $2 \mathbf{b}(2.29 \mathrm{~g}, 10 \mathrm{mmol})$ and $N, N$-dimethylformamide dimethyl acetal $(1.19 \mathrm{~g}, 10 \mathrm{mmol})$, it was obtained compound $3 \mathbf{b}(58 \%)$ as yellow solid, $\operatorname{mp} 156^{\circ} \mathrm{C}$. IR $\left(v_{\max } / \mathrm{cm}^{-1}\right): 1505(\mathrm{C}=\mathrm{C})$, $1608(\mathrm{C}=\mathrm{C}), 1680(\mathrm{C}=\mathrm{O}), 2197(\mathrm{CN}) .{ }^{1} \mathrm{H}$ NMR $\left(400 \mathrm{MHz}, \mathrm{DMSO}_{-} \mathrm{d}_{6}\right): \delta_{\mathrm{H}} 1.22(3 \mathrm{H}, \mathrm{t}, J=7.1$ $\left.\mathrm{Hz}, \mathrm{CH}_{2}-\mathrm{CH}_{3}\right), 2.36\left(3 \mathrm{H}, \mathrm{s}, \mathrm{Ph}-\mathrm{CH}_{3}\right), 2.97\left(3 \mathrm{H}, \mathrm{s}, \mathrm{NCH}_{3}\right), 3.02\left(3 \mathrm{H}, \mathrm{s}, \mathrm{NCH}_{3}\right), 4.13(2 \mathrm{H}, \mathrm{q}, J$ $\left.=7.1 \mathrm{~Hz}, \mathrm{CH}_{2}-\mathrm{CH}_{3}\right), 6.66(1 \mathrm{H}, \mathrm{d}, J=12.6 \mathrm{~Hz}, \mathrm{CH}=\mathrm{CH}-\mathrm{N}), 7.25(1 \mathrm{H}, \mathrm{d}, J=12.6 \mathrm{~Hz}$, $\mathrm{CH}=\mathrm{CH}-\mathrm{N}), 7.08-7.24\left(\mathrm{~m}, 4 \mathrm{H}_{\text {arom }}\right),{ }^{13} \mathrm{C} \mathrm{NMR}\left(101 \mathrm{MHz}, \mathrm{DMSO}-\mathrm{d}_{6}\right): \delta_{\mathrm{C}} 14.9\left(\mathrm{CH}_{2} \mathrm{CH}_{3}\right), 38.0$ $\left(\mathrm{NCH}_{3}\right), 21.4\left(\mathrm{Ph}_{-} \mathrm{CH}_{3}\right), 45.8\left(\mathrm{NCH}_{3}\right), 59.8\left(\mathrm{CH}_{2}-\mathrm{CH}_{3}\right), 82.9(\mathrm{C}=\mathrm{C}(\mathrm{CN}), 99.7(\mathrm{C}=\mathrm{C}-\mathrm{N}), 120.4$ $(\mathrm{CN}), 129.0-138.5\left(6 \mathrm{C}_{\text {arom }}\right), 158.0(\mathrm{C}=\mathrm{C}-\mathrm{N}), 165.3(\mathrm{C}=\mathrm{O}), 169.17[\mathrm{C}=\mathrm{C}(\mathrm{CN})]$.Calcd. For $\mathrm{C}_{17} \mathrm{H}_{20} \mathrm{~N}_{2} \mathrm{O}_{2}: 71.81 \mathrm{C}, 7.09 \mathrm{H}, 9.85$. Found: $71.89 \mathrm{C}, 7.26 \mathrm{H}, 10.77 \mathrm{~N}$.

\section{Ethyl 2-cyano-3-(2,4-dichlorophenyl)-5-dimethylaminopenta-2,4-dienoate 3c}

From $2 \mathrm{c}(2.84 \mathrm{~g}, 10 \mathrm{mmol})$ and $N, N$-dimethylformamide dimethyl acetal $(1.19 \mathrm{~g}, 10 \mathrm{mmol})$, it was obtained compound $3 \mathbf{c}(70 \%)$ as yellow solid, mp $168^{\circ} \mathrm{C}$. IR $\left(v_{\max } / \mathrm{cm}^{-1}\right): 1509(\mathrm{C}=\mathrm{C})$, $1607(\mathrm{C}=\mathrm{C}), 1682(\mathrm{C}=\mathrm{O}), 220.1(\mathrm{CN}) .{ }^{1} \mathrm{H}$ NMR $\left(\mathrm{DMSO}_{-} \mathrm{d}_{6}\right): \delta_{\mathrm{H}} 1.22(3 \mathrm{H}, \mathrm{t}, J=7.10 \mathrm{~Hz}$, $\left.\mathrm{CH}_{2}-\mathrm{CH}_{3}\right), 2.99\left(3 \mathrm{H}, \mathrm{s}, \mathrm{NCH}_{3}\right), 3.08\left(3 \mathrm{H}, \mathrm{s}, \mathrm{NCH}_{3}\right), 4.13\left(2 \mathrm{H}, \mathrm{q}, \mathrm{J}=7.1 \mathrm{~Hz}, \mathrm{CH}_{2}-\mathrm{CH}_{3}\right) .5 .75$ $(1 \mathrm{H}, \mathrm{d}, J=12.8 \mathrm{~Hz}, \mathrm{CH}=\mathrm{CH}-\mathrm{N}), 6.66(1 \mathrm{H}, \mathrm{d}==12,8 \mathrm{~Hz}, \mathrm{CH}=\mathrm{CH}-\mathrm{N}) 7,10-7,39(3 \mathrm{H}, \mathrm{m}$, $\left.\mathrm{H}_{\text {arom }}\right),{ }^{13} \mathrm{C}$ NMR $\left(101 \mathrm{MHz}, \mathrm{DMSO}-\mathrm{d}_{6}\right): \delta_{\mathrm{C}} 14.5\left(\mathrm{CH}_{2} \mathrm{CH}_{3}\right), 38.1\left(\mathrm{NCH}_{3}\right), 45.7\left(\mathrm{NCH}_{3}\right), 60.1$ $\left(\mathrm{CH}_{2} \mathrm{CH}_{3}\right), 82.8(\mathrm{C}=\mathrm{C}(\mathrm{CN})), 98.8(\mathrm{C}=\mathrm{C}-\mathrm{N}), 119.7(\mathrm{CN}), 127.5-135.2\left(6 \mathrm{C}_{\text {arom }}\right), 136.2(\mathrm{C}=\mathrm{C}-$ $\mathrm{N}), 164.4(\mathrm{C}=\mathrm{O}), 165.1(\mathrm{C}=\mathrm{C}(\mathrm{CN}))$.Calcd. for $\mathrm{C}_{16} \mathrm{H}_{16} \mathrm{Cl}_{2} \mathrm{~N}_{2} \mathrm{O}_{2}: 56.65 \mathrm{C}, 4.75 \mathrm{H}, 8.26 \mathrm{~N}$. Found: $57.07 \mathrm{C}, 4.70 \mathrm{H}, 8.16 \mathrm{~N}$.

\section{Ethyl 2-cyano-5-dimethylamino-3-(4-nitrophenyl)penta-2,4 dienoate 3d}

From $(2.60 \mathrm{~g}, 10 \mathrm{mmol})$ of 2 dand $N, N$-dimethylformamide dimethyl acetal $(1.19 \mathrm{~g}, 10 \mathrm{mmol})$, it was obtained compound $\mathbf{3 d}(54 \%)$ as yellow solid, mp $185^{\circ} \mathrm{C}$. IR $\left(v_{\max } / \mathrm{cm}^{-1}\right): 1509(\mathrm{C}=\mathrm{C})$, $1607(\mathrm{C}=\mathrm{C}) 1682(\mathrm{C}=\mathrm{O}), 2201(\mathrm{CN}) .{ }^{1} \mathrm{H}$ NMR $\left(400 \mathrm{MHz}, \mathrm{DMSO}-\mathrm{d}_{6}\right): \delta_{\mathrm{H}} 1.22(3 \mathrm{H}, \mathrm{t}, J=7.10$ $\left.\mathrm{Hz}, \mathrm{CH}_{2}-\mathrm{CH}_{3}\right), 3.00\left(3 \mathrm{H}, \mathrm{s}, \mathrm{NCH}_{3}\right), 3.05\left(3 \mathrm{H}, \mathrm{s}, \mathrm{NCH}_{3}\right), 4.15\left(2 \mathrm{H}, \mathrm{q}, J=7.1 \mathrm{~Hz}, \mathrm{CH}_{2}-\mathrm{CH}_{3}\right)$, $6.67(1 \mathrm{H}, \mathrm{d}, J=12.8 \mathrm{~Hz}, \mathrm{CH}=\mathrm{CH}-\mathrm{N}), 7.12(1 \mathrm{H}, \mathrm{d}, J=12.8 \mathrm{~Hz}, \mathrm{CH}=\mathrm{CH}-\mathrm{N}), 7.51(2 \mathrm{H}, \mathrm{d}, \mathrm{H}$ arom), $8.28\left(2 \mathrm{H}, \mathrm{d}, \mathrm{H}_{\text {arom }}\right) .{ }^{13} \mathrm{C}$ NMR (101 MHz, DMSO-d $\left.{ }_{6}\right): \delta_{\mathrm{C}} 14.8\left(\mathrm{CH}_{2} \mathrm{CH}_{3}\right), 38.1\left(\mathrm{NCH}_{3}\right)$, 
$45.7\left(\mathrm{NCH}_{3}\right), 60.1\left(\mathrm{CH}_{2} \mathrm{CH}_{3}\right), 82.4(\mathrm{C}=\mathrm{C}(\mathrm{CN})), 99.4(\mathrm{C}=\mathrm{C}-\mathrm{N}), 120.0(\mathrm{CN}), 124.0-146.3(6$ $\left.\mathrm{C}_{\text {arom }}\right), 148.1(\mathrm{C}=\mathrm{C}-\mathrm{N}), 164.9(\mathrm{C}=\mathrm{O}), 166.4[\mathrm{C}=\mathrm{C}(\mathrm{CN})] . \mathrm{Calcd}$. for $\mathrm{C}_{16} \mathrm{H}_{17} \mathrm{~N}_{3} \mathrm{O}_{4}: 60.94 \mathrm{C}, 5.43$ $\mathrm{H} ; 13.33$ N. Found: $60.91 \mathrm{C}, 5.30 \mathrm{H}, 13.43 \mathrm{~N}$.

A mixture of enaminonitriles 3a-d $(20 \mathrm{mmol})$, primary diamines $(10 \mathrm{mmol})$ were dissolved in DMF $(20 \mathrm{~mL})$ and heated for a 24 hours. The progress of the reaction was monitored by TLC (n-Hexane/Ethyl acetate $=2 / 8$ ) to make sure about the completion of the reaction. After cooling, the white solid product was isolated through filtration and thorough washing with diethyl ether to give bis-2-pyridone derivatives $\mathbf{4 a - j}$.

\section{1-[(3-Cyano-2-oxo-4-phenylpyridin-1(2H)-yl) methyl]-2-oxo-4-phenyl-1,2-dihydro- pyridine-3-carbonitrile 4a:}

From $(0.54 \mathrm{~g}, 20 \mathrm{mmol})$ of $\mathbf{3 b}$ and of 1, 2-ethylenediamine $(0.06 \mathrm{~g}, 10 \mathrm{mmol})$ it was obtained compound 3c $(57 \%)$ as yellow solid, $\mathrm{mp}=249{ }^{\circ} \mathrm{C}$. IR $\left(v_{\max } / \mathrm{cm}^{-1}\right): 1609(\mathrm{C}=\mathrm{C}), 1653(\mathrm{C}=\mathrm{C})$, $1683(\mathrm{C}=\mathrm{O}), 2192(\mathrm{CN}) .{ }^{1} \mathrm{H}$ NMR $\left(400 \mathrm{MHz}, \mathrm{DMSO}-\mathrm{d}_{6}\right): \delta_{\mathrm{H}} 4.37-4.39\left(4 \mathrm{H}, \mathrm{s}, 2 \mathrm{CH}_{2}\right), 6.49$ $(2 \mathrm{H}, \mathrm{d}, J=7.0 \mathrm{~Hz}, 2 \mathrm{CH}=\mathrm{CH}-\mathrm{N}), 7.51-7.65\left(10 \mathrm{H}, \mathrm{m}, \mathrm{H}_{\text {arom }}\right), 7.97(2 \mathrm{H}, \mathrm{d}, J=7.0 \mathrm{~Hz}, 2$ $\mathrm{CH}=\mathrm{CH}-\mathrm{N}),{ }^{13} \mathrm{C}$ NMR $\left(101 \mathrm{MHz}, \mathrm{DMSO}-\mathrm{d}_{6}\right): \delta_{\mathrm{C}} 48.6\left(\mathrm{CH}_{2}\right), 100.8(\mathrm{C}=\mathrm{C}(\mathrm{CN})), 107.3(\mathrm{C}=\mathrm{C}-$ $\mathrm{N}), \quad 116.7 \quad(\mathrm{CN}), \quad 128.7-136.0 \quad\left(6 \times \mathrm{C}_{\text {arom }}\right), \quad 144.3 \quad(\mathrm{C}=\mathrm{C}-\mathrm{N}), \quad 160.1 \quad(\mathrm{C}=\mathrm{O})$, 160.6[C $=\mathrm{C}(\mathrm{CN})]$.Calcd for $\mathrm{C}_{26} \mathrm{H}_{18} \mathrm{~N}_{4} \mathrm{O}_{2}: 74.63 \mathrm{C}, 4.34 \mathrm{H}, 13.39 \mathrm{~N}$. Found: $75.16 \mathrm{~N}, 4.30 \mathrm{H}$, $13.46 \mathrm{~N}$.

\section{1-[3-(3-Cyano-2-oxo-4-phenylpyridin-1(2H)-yl)propyl]-2-oxo-4-phenyl-1,2-dihydro- pyridine-3-carbonitrile $4 \mathrm{~b}$ :}

From 3b $(0.54 \mathrm{~g}, 20 \mathrm{mmol})$ and butane-1,4-diamine $(0.08 \mathrm{~g}, 10 \mathrm{mmol})$ it was obtained compound $4 \mathbf{b}(60 \%), \mathrm{mp}=220{ }^{\circ} \mathrm{C}$ as white solid. IR $\left(v_{\max } / \mathrm{cm}^{-1}\right): 1512(\mathrm{C}=\mathrm{C}), 1600(\mathrm{C}=\mathrm{C})$, $1645(\mathrm{C}=\mathrm{O}), 2221(\mathrm{CN}) .{ }^{1} \mathrm{H}$ NMR $\left(400 \mathrm{MHz}, \mathrm{DMSO}-\mathrm{d}_{6}\right): \delta_{\mathrm{H}} 1.71-1.80\left(4 \mathrm{H}, \mathrm{s}, 2 \mathrm{CH}_{2}\right), 4.00-$ $4.08\left(4 \mathrm{H}, \mathrm{s}, 2 \mathrm{CH}_{2}\right), 6.51(2 \mathrm{H}, \mathrm{d}, J=7.0 \mathrm{~Hz}, 2 \mathrm{CH}=\mathrm{CH}-\mathrm{N}), 7.51-7.65\left(10 \mathrm{H}, \mathrm{m}, \mathrm{H}_{\text {arom }}\right), 8.13$ $(2 \mathrm{H}, \mathrm{d}, J=7.0 \mathrm{~Hz}, 2 \mathrm{CH}=\mathrm{CH}-\mathrm{N}),{ }^{13} \mathrm{C}$ NMR $\left(101 \mathrm{MHz}, \mathrm{DMSO}-\mathrm{d}_{6}\right): \delta_{\mathrm{C}} 25.9\left(\mathrm{C}_{-} \mathrm{CH}_{2}-\mathrm{CH}_{2}\right)$, $49.5\left(\mathrm{C}-\mathrm{CH}_{2}-\mathrm{CH}_{2}\right), 100.8(\mathrm{C}=\mathrm{C}(\mathrm{CN})), 107.2(\mathrm{C}=\mathrm{C}-\mathrm{N}), 116.8(\mathrm{CN}), 128.6-136.0\left(6 \mathrm{C}_{\text {arom }}\right)$, $144.3(\mathrm{C}=\mathrm{C}-\mathrm{N}), 159.8(\mathrm{C}=\mathrm{O}), 160.4(\mathrm{C}=\mathrm{C}(\mathrm{CN}))$. Calcd. for $\mathrm{C}_{28} \mathrm{H}_{22} \mathrm{~N}_{4} \mathrm{O}_{2}: 75.3 \mathrm{C}, 4.97 \mathrm{H}$, 12.55 N. Found: $75.36 \mathrm{C}, 4.89 \mathrm{H}, 12.75 \mathrm{~N}$.

\section{1-[3-(3-yano-2-oxo-4-phenylpyridin-1(2H)-yl)propyl]-2oxo-4-phenyl-1,2-dihydropyri- dine -3-carbonitrile $4 \mathrm{c}$ :}

From 3b $(0.54 \mathrm{~g}, 20 \mathrm{mmol})$ and hexane-1,6-diamine $(0.11 \mathrm{~g}, 10 \mathrm{mmol})$ it was obtained compound $4 \mathbf{c}(54 \%), \mathrm{mp} 251{ }^{\circ} \mathrm{C}$ as white solid. IR $\left(v_{\max } / \mathrm{cm}^{1}\right) 1509(\mathrm{C}=\mathrm{C}), 1623(\mathrm{C}=\mathrm{C}), 1690$ $(\mathrm{C}=\mathrm{O}), 2223(\mathrm{CN}) .{ }^{1} \mathrm{H}$ NMR $\left(400 \mathrm{MHz}, \mathrm{DMSO}-\mathrm{d}_{6}\right): \delta_{\mathrm{H}} 1.29-1.42\left(4 \mathrm{H}, \mathrm{s}, 2 \mathrm{CH}_{2}\right), 1.64-1.77$ $\left(4 \mathrm{H}, \mathrm{s}, 2 \mathrm{CH}_{2}\right), 3.92-4.08\left(4 \mathrm{H}, \mathrm{s}, 2 \mathrm{CH}_{2}\right), 6.53(2 \mathrm{H}, \mathrm{d}, J=7.0 \mathrm{~Hz}, 2 \mathrm{CH}=\mathrm{CH}-\mathrm{N}), 7.53-7.68$ $\left(10 \mathrm{H}, \mathrm{m}, \mathrm{H}_{\text {arom }}\right), 8.15(2 \mathrm{H}, \mathrm{d}, J=7.0 \mathrm{~Hz}, 2 \mathrm{CH}=\mathrm{CH}-\mathrm{N}) .{ }^{13} \mathrm{C}$ NMR $\left(101 \mathrm{MHz}, \mathrm{DMSO}-\mathrm{d}_{6}\right): \delta_{\mathrm{C}}$

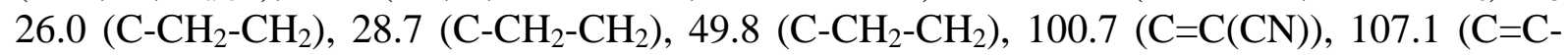
$\mathrm{N}), 116.8(\mathrm{CN}), 128.5-136.0\left(6 \mathrm{C}_{\text {arom }}\right), 144.2(\mathrm{C}=\mathrm{C}-\mathrm{N}), 159.7(\mathrm{C}=\mathrm{O}), 160.3(\mathrm{C}=\mathrm{C}(\mathrm{CN}))$. Calcd. $\mathrm{C}_{30} \mathrm{H}_{26} \mathrm{~N}_{4} \mathrm{O}_{2}: 75.93 \mathrm{C}, 5.52 \mathrm{H}, 11.61 \mathrm{~N}$. Found: $75.82 \mathrm{C}, 5.52 \mathrm{H}, 11.68 \mathrm{~N}$. 


\section{1-[(3-Cyano-2-oxo-4-p-tolylpyridin-1(2H)-yl)methyl]-2-oxo-4-p-tolyl-1,2-dihydro- pyridine-3-carbonitrile 4d:}

From $3 \mathbf{b}(0.58 \mathrm{~g}, 20 \mathrm{mmol})$ and 1,2-ethylenediamine $(0.06 \mathrm{~g}, 10 \mathrm{mmol})$, it was obtained compound $4 \mathbf{d}(46 \%), \mathrm{mp}=235^{\circ} \mathrm{C}$ as white solid. IR $\left(v_{\max } / \mathrm{cm}^{1}\right): 1509(\mathrm{C}=\mathrm{C}), 1598(\mathrm{C}=\mathrm{C})$, $1652(\mathrm{C}=\mathrm{O}), 2221(\mathrm{CN}) .{ }^{1} \mathrm{H}$ NMR $\left(400 \mathrm{MHz}, \mathrm{DMSO}-\mathrm{d}_{6}\right): \delta_{\mathrm{H}} 2.39\left(6 \mathrm{H}, \mathrm{s}, 2 \mathrm{Ph}-\mathrm{CH}_{3}\right), 4.34-$ $4.36\left(4 \mathrm{H}, \mathrm{m}, 2 \mathrm{CH}_{2}\right), 6.49(2 \mathrm{H}, \mathrm{d}, J=7.0 \mathrm{~Hz}, 2 \mathrm{CH}=\mathrm{CH}-\mathrm{N}), 7.34-7.58\left(10 \mathrm{H}, \mathrm{m}, \mathrm{H}_{\text {arom }}\right), 7.96$ $(2 \mathrm{H}, \mathrm{d}, J=7.0 \mathrm{~Hz}, 2 \mathrm{CH}=\mathrm{CH}-\mathrm{N}),{ }^{13} \mathrm{C}$ NMR $\left(400 \mathrm{MHz}, \mathrm{DMSO}-\mathrm{d}_{6}\right): \delta_{\mathrm{C}} 21.4\left(\mathrm{Ph}_{-} \mathrm{CH}_{3}\right), 45.4$ $\left(\mathrm{N}-\mathrm{CH}_{2}-\mathrm{CH}_{2}\right), 100.6(\mathrm{C}=\mathrm{C}(\mathrm{CN})), 106.9(\mathrm{C}=\mathrm{C}-\mathrm{N}), 116.5(\mathrm{CN}), 128.5-141.3\left(6 \mathrm{C}_{\text {arom }}\right), 144.2$ $(\mathrm{C}=\mathrm{CN}), 158.5(\mathrm{C}=\mathrm{O}), 160.7(\mathrm{C}=\mathrm{C}(\mathrm{CN}))$. Calcd. for $\mathrm{C}_{28} \mathrm{H}_{22} \mathrm{~N}_{4} \mathrm{O}_{2}: 75.32 \mathrm{C}, 4.97 \mathrm{H}, 12.55 \mathrm{~N}$. Found: $73.69 \mathrm{C}, 4.95 \mathrm{H}, 12.57 \mathrm{~N}$.

\section{1-[3-(3-Cyano-2-oxo-4-p-tolylpyridin-1(2H)-yl)propyl]-2oxo-4-p-tolyl-1,2-dihydro- pyridine-3-carbonitrile 4e:}

From 3b (0.56 g, $20 \mathrm{mmol})$ and butane-1,4-diamine $(0.08 \mathrm{~g}, 10 \mathrm{mmol})$ it was obtained compound $4 \mathbf{e}(70 \%), \mathrm{mp}=264{ }^{\circ} \mathrm{C}$ as white solid. IR $\left(v_{\max } / \mathrm{cm}^{-1}\right): 1506(\mathrm{C}=\mathrm{C}), 1596(\mathrm{C}=\mathrm{C})$, $1652(\mathrm{C}=\mathrm{O}), 2218(\mathrm{CN}) .{ }^{1} \mathrm{H}$ NMR $\left(400 \mathrm{MHz}, \mathrm{DMSO}-\mathrm{d}_{6}\right): \delta_{\mathrm{H}} 1.70-1.76\left(\mathrm{~N}^{-C_{2}}-\mathrm{CH}_{2}\right), 2.39$ $\left(6 \mathrm{H}, \mathrm{s}, 2 \mathrm{Ph}-\mathrm{CH}_{3}\right), 3.99-4.06\left(4 \mathrm{H}, \mathrm{m}, 2 \mathrm{CH}_{2}\right), 6.52(2 \mathrm{H}, \mathrm{d}, J=7.0 \mathrm{~Hz}, 2 \mathrm{CH}=\mathrm{CH}-\mathrm{N}), 7.34-$ $7.58\left(10 \mathrm{H}, \mathrm{m}, \mathrm{H}_{\text {arom }}\right), 8.14(2 \mathrm{H}, \mathrm{d}, J=7.0 \mathrm{~Hz}, 2 \mathrm{CH}=\mathrm{CH}-\mathrm{N}) .{ }^{13} \mathrm{C}$ NMR (400 MHz, DMSO-

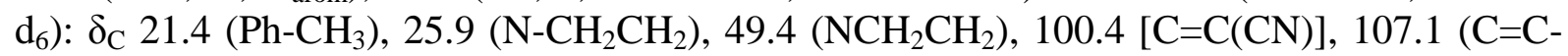
$\mathrm{N}), 116.9(\mathrm{CN}), 128.5-141.1\left(6 \mathrm{C}_{\text {arom }}\right), 144.1(\mathrm{C}=\mathrm{C}-\mathrm{N}), 159.7(\mathrm{C}=\mathrm{O}), 160.5[\mathrm{C}=\mathrm{C}(\mathrm{CN})]$. Calcd. for $\mathrm{C}_{30} \mathrm{H}_{26} \mathrm{~N}_{4} \mathrm{O}_{2}: 75.93 \mathrm{C}, 5.52 \mathrm{H}, 11.81 \mathrm{~N}$. Found: $76.15 \mathrm{C}, 5.54 \mathrm{H}, 11.71 \mathrm{~N}$.

\section{1-[3-(3-Cyano-4-(4-nitrophenyl)-2-oxopyridin-1(2H)-yl)propyl]-4-(4-nitrophenyl)-2-oxo- 1,2-dihydropyridine-3-carbonitrile 4j:}

From $3 \mathbf{d}(0.70 \mathrm{~g}, 20 \mathrm{mmol})$ and butane-1,4-diamine $(0.08 \mathrm{~g}, 10 \mathrm{mmol})$ it was obtained compound $4 \mathbf{j}(62 \%), \mathrm{mp}=257{ }^{\circ} \mathrm{C}$ as white solid. $\mathrm{IR}\left(v_{\max } / \mathrm{cm}^{-1}\right): 1521(\mathrm{C}=\mathrm{C}), 1600(\mathrm{C}=\mathrm{C})$, $1653(\mathrm{C}=\mathrm{O}), 2224(\mathrm{CN}) .{ }^{1} \mathrm{H}$ NMR $\left(400 \mathrm{MHz}, \mathrm{DMSO}-\mathrm{d}_{6}\right): \delta_{\mathrm{H}} 1.73-1.79\left(4 \mathrm{H}, \mathrm{m}, 2 \mathrm{CH}_{2}\right), 4.03-$ $4.09\left(4 \mathrm{H}, \mathrm{m}, 2 \mathrm{CH}_{2}\right), 6.60(2 \mathrm{H}, \mathrm{d}, J=7.0 \mathrm{~Hz}, 2 \mathrm{CH}=\mathrm{CH}-\mathrm{N}), 7.90-7.94\left(4 \mathrm{H}, \mathrm{m}, \mathrm{H}_{\text {arom }}\right), 8.24$ $(2 \mathrm{H}, \mathrm{d}, J=7.0 \mathrm{~Hz}, 2 \mathrm{CH}=\mathrm{CH}-\mathrm{N}), 8.37-8.41\left(4 \mathrm{H}, \mathrm{m}, \mathrm{H}_{\text {arom }}\right),{ }^{13} \mathrm{C} \mathrm{NMR}\left(101 \mathrm{MHz}, \mathrm{DMSO}-\mathrm{d}_{6}\right)$ :

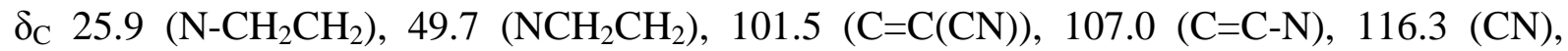
124.4-144.9 $\left(\mathrm{C}_{\text {arom }}\right), 149.0(\mathrm{C}=\mathrm{C}-\mathrm{N}), 157.8(\mathrm{C}=\mathrm{O}), 160.1(\mathrm{C}=\mathrm{C}(\mathrm{CN}))$. Calcd. for $\mathrm{C}_{28} \mathrm{H}_{20} \mathrm{~N}_{6} \mathrm{O}_{6}$ : 62.68 C, 3.76 H, 15.66 N. Found: 62.48 C, 3.86 H, 15.66 N.

\section{1-[3-(3-Cyano-4-(4-nitrophenyl)-2-oxopyridin-1(2H)-yl)propyl]-4-(4-nitrophenyl)-2-oxo- 1,2-dihydropyridine-3-carbonitrile 4k:}

From 3d $(0.70 \mathrm{~g}, 20 \mathrm{mmol})$ and hexane-1,6-diamine $(0.11 \mathrm{~g}, 10 \mathrm{mmol})$ it was obtained compound $4 \mathbf{k}(49 \%), \mathrm{mp}=259^{\circ} \mathrm{C}$ as white solid. IR $\left(v_{\max } / \mathrm{cm}^{-1}\right): 1522(\mathrm{C}=\mathrm{C}), 1603(\mathrm{C}=\mathrm{C})$, $1646(\mathrm{C}=\mathrm{O}), 2222(\mathrm{CN}) .{ }^{1} \mathrm{H}$ NMR $\left(400 \mathrm{MHz}, \mathrm{DMSO}-\mathrm{d}_{6}\right) \delta_{\mathrm{H}} 1.20-1.27\left(4 \mathrm{H}, \mathrm{m}, 2 \mathrm{CH}_{2}\right), 1.72-$ $1.81\left(4 \mathrm{H}, \mathrm{m}, 2 \mathrm{CH}_{2}\right), 4.03-4.10\left(4 \mathrm{H}, \mathrm{m}, 2 \mathrm{CH}_{2}\right), 6.60(2 \mathrm{H}, \mathrm{d}, J=7.0 \mathrm{~Hz}, 2 \mathrm{CH}=\mathrm{CH}-\mathrm{N}), 7.89-$ $7.93\left(4 \mathrm{H}, \mathrm{m}, \mathrm{H}_{\text {arom }}\right), 8.24(2 \mathrm{H}, \mathrm{d}, J=7.0 \mathrm{~Hz}, 2 \mathrm{CH}=\mathrm{CH}-\mathrm{N}), 8.36-8.43\left(4 \mathrm{H}, \mathrm{m}, \mathrm{H}_{\text {arom }}\right),{ }^{13} \mathrm{C}$

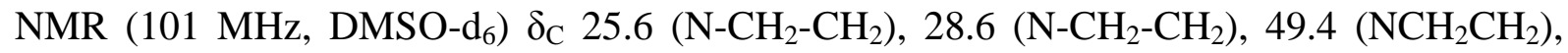
$101.0(\mathrm{C}=\mathrm{C}(\mathrm{CN})), 106.5(\mathrm{C}=\mathrm{CN}), 116.3(\mathrm{CN}), 130.2\left(6 \mathrm{C}_{\text {arom }}\right), 142.1(\mathrm{C}=\mathrm{CN}), 145.0(\mathrm{C}=\mathrm{O})$, $159.7(\mathrm{C}=\mathrm{C}(\mathrm{CN}))$. Calcd for $\mathrm{C}_{30} \mathrm{H}_{24} \mathrm{~N}_{6} \mathrm{O}_{6}: 63.82 \mathrm{C}, 4.28 \mathrm{H}, 14.89 \mathrm{~N}$. Found: $62.64 \mathrm{C}, 3.79 \mathrm{H}$, $15.35 \mathrm{~N}$.

\section{Crystallographic data:}

Crystal data for 3a: $\mathrm{C}_{16} \mathrm{H}_{18} \mathrm{~N}_{2} \mathrm{O}_{2}, M=270.32$, monoclinic, space group $I 2 / \mathrm{a}, Z=8, a=$ $14.898(2) \AA, b=13.702(2) \AA, c=14.929(2) \AA, \beta=109.276(3)^{\circ}, V=2876.8(7) \AA^{3}, \mu(\mathrm{Mo}-$ $\mathrm{K} \alpha)=0.083 \mathrm{~mm}^{-1}, D_{\mathrm{c}}=1.248 \mathrm{~g} \mathrm{~cm}^{-3}$, yellow block with crystal size of $0.19 \times 0.19 \times 0.17 \mathrm{~mm}^{3}$. Of a total of 28362 reflections collected, 2647 were independent $\left(R_{\text {int }}=0.070\right)$. Final 
$R 1=0.0432[I>2 \sigma(I)]$ and $w R 2=0.1105$ (all data). Data completeness to theta $=25.24^{\circ}$, 100\%. CCDC1943247

Crystal data for $4 \mathrm{c}: \mathrm{C}_{15} \mathrm{H}_{13} \mathrm{~N}_{2} \mathrm{O}, M=237.27$, monoclinic, space group $P 2{ }_{1} / \mathrm{c}, Z=4, a=$

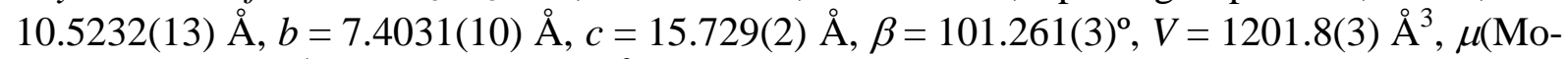
$\mathrm{K} \alpha)=0.08 \mathrm{~mm}^{-1}, D_{\mathrm{c}}=1.311 \mathrm{~g} \mathrm{~cm}^{-3}$, colourless plate with crystal size of $0.14 \times 0.12 \times 0.04$ $\mathrm{mm}^{3}$. Of a total of 8279 reflections collected, 2207 were independent $\left(R_{\mathrm{int}}=0.034\right)$. Final $R 1=0.0351[I>2 \sigma(I)]$ and $w R 2=0.0955$ (all data). Data completeness to theta $=25.4^{\circ}$, 99.9\%. CCDC1936900.

\section{Results and discussion}

The synthesis of bis-2-pyridone derivatives was based on three steps, the first and the second ones were previously published by our group [45]. First we have prepared easily $\alpha, \beta-$ unsaturated compounds 2a-c in moderate yields, via a Knoevenagel condensation, of aromatic ketones with ethyl cyanoacetate, catalyzed by ammonium acetate, under solvent-free conditions (Table 1).

Table 1. Synthesis of olefins derivatives 2a-c

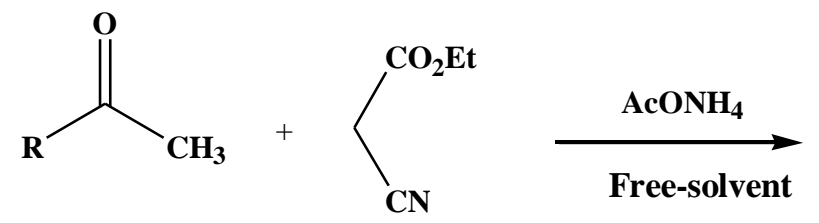

1a-c<smiles>[R]C(C)=C(C#N)C(=O)OC</smiles>

2a-c

\begin{tabular}{cccc}
\hline Entry & R & Product & Yield (\%) \\
\hline 1 & $\mathrm{C}_{6} \mathrm{H}_{5^{-}}$ & $2 \mathrm{a}$ & 47 \\
2 & $4-\mathrm{Me}_{6} \mathrm{H}_{4}-$ & $2 \mathrm{~b}$ & 45 \\
3 & $2,4-\mathrm{Cl}^{-} \mathbf{C}_{6} \mathrm{H}_{3^{-}}$ & $2 \mathrm{c}$ & 50 \\
4 & $4-\mathrm{NO}_{2}-\mathrm{C}_{6} \mathrm{H}^{-}$ & $2 \mathrm{~d}$ & 62 \\
\hline
\end{tabular}

The second step is the formation of enaminonitriles3a-d as key intermediate scaffolds from olefins 2a-c. Enaminonitriles are known to be very reactive intermediates and serves as pushpull dienes. Here we have prepared enaminonitriles 3a-d by reacting olefins $\mathbf{2 a - d}$ with one equivalent of $N, N$-dimethylformamide dimethyl acetal (DMFDMA) under mild solvent free conditions, at ambient temperature for $2 \mathrm{~h}$ (Table 2). 
Table 2. Synthesis of enaminonitriles 3a-d derivatives<smiles>[R]C(C)=C(C#N)C(=O)OCC</smiles>

2a-d<smiles>COC(OC)N(C)[14CH3]</smiles>

OMe

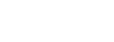

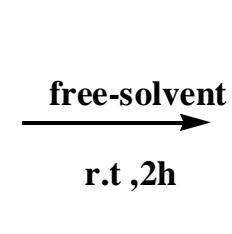<smiles>[R]C(/C=C/N(C)C)=C(\C#N)C(=O)OC</smiles>

3a-d

\begin{tabular}{lccc}
\hline Entry & $\mathrm{R}$ & Product & Yield (\%) \\
\hline 1 & $\mathrm{C}_{6} \mathrm{H}_{5^{-}}$ & $3 \mathrm{a}$ & 69 \\
2 & $4-\mathrm{Me}_{-} \mathrm{H}_{4}-$ & $3 \mathrm{~b}$ & 58 \\
3 & $2,4-\mathrm{Cl}_{6} \mathrm{C}_{6} \mathrm{H}_{3^{-}}$ & $3 \mathrm{c}$ & 70 \\
4 & $4-\mathrm{NO}_{2}-\mathrm{C}_{6} \mathbf{H}^{-}$ & $3 \mathrm{~d}$ & 54 \\
\hline
\end{tabular}

The structure of compound 3aas a key intermediate was firstly characterized by spectroscopic analysis and by single-crystal X-ray diffraction analysis (Figure 3).

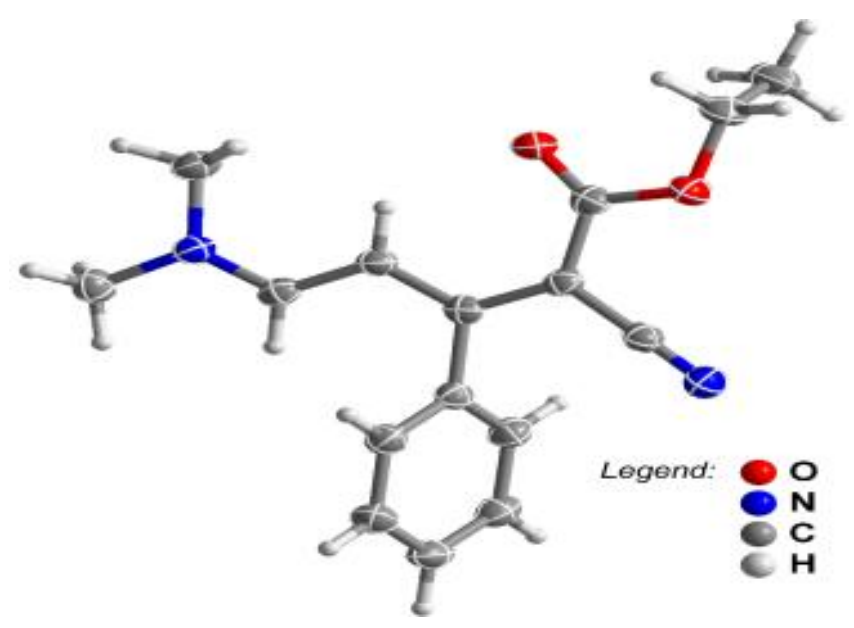

Figure 3. Molecular unit present in compound 3a

Compound 3a crystallizes in the centrosymmetric monoclinic $I \mathrm{a} / 2$ space group with the asymmetric unit being composed of a sole enaminonitrile molecular unit as depicted in Figure 3. The molecule close packs mediated mainly by weak hydrogen bonding interactions; of the $\mathrm{C}-\mathrm{H} \cdots \mathrm{O}$ type, with the refined $\mathrm{d}_{\mathrm{C} \cdots \mathrm{O}}$ interatomic distances being found in the $2.885(2)$ $3.369(3) \AA$ range, having $<(\mathrm{CHO})$ interaction angles in the $126-155^{\circ}$ interval; and of the $\mathrm{C}-\mathrm{H} \cdots \mathrm{N}$ type, with the refined $\mathrm{d}_{\mathrm{C} \cdots \mathrm{N}}$ interatomic distances found in the 3.478(3)-3.572(3) $\AA$ 
range having $<(\mathrm{CHO})$ interaction angles in the $168-171^{\circ}$ interval. Despite the presence of an aromatic ring, no structurally significant $\pi-\pi$ interaction are present in the crystal structure. The final step of this synthesis is the bis-2-pyridone ring formation, through the reaction of 3a-d with various diamines in the presence of DMF, at reflux, in 49-85\% yield (Table 3).

Table 3. Structures and yields of the cyclization to bis-2-pyridones $\mathbf{4 a - k}$

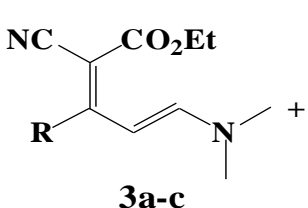<smiles>[R]c1ccn(C2CC2n2ccc([R])c(C#N)c2=O)c(=O)c1C#N</smiles>

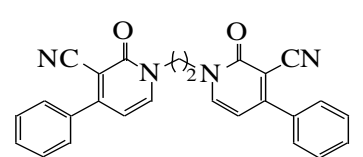

4a, $57 \%$<smiles>Cc1ccc(C2=C(C#N)C(=O)C(Nn3ccc(-c4ccc(C)cc4)c(C#N)c3=O)C=C2)cc1</smiles>

4d, $64 \%$

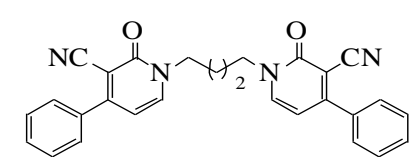

$4 b, 60 \%$

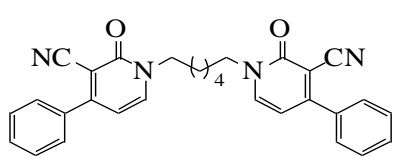

4c, $54 \%$<smiles>N#CC1C(=O)C(NCCNCN2CCC(C3CCC(Cl)CC3Cl)C(C#N)C2=O)CCC1C1CCC(Cl)CC1Cl</smiles>

4g, $77 \%$

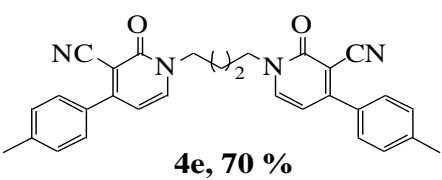

4e, $70 \%$

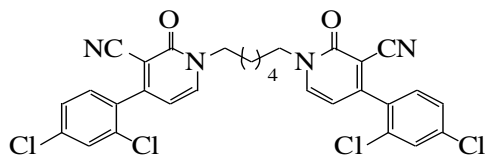

4h, $85 \%$

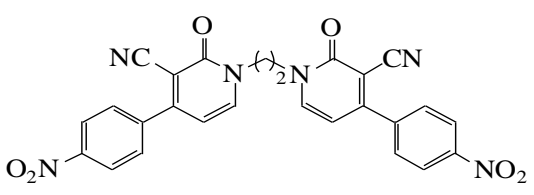

4i, $58 \%$

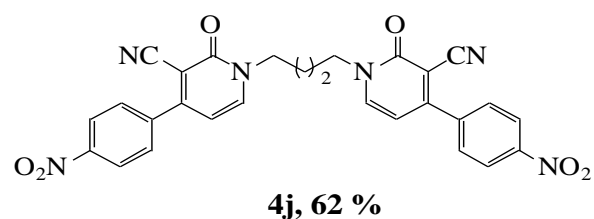

$4 \mathbf{j}, 62 \%$<smiles>N#CC1C(=O)N(CCN2CCC(C3CCC([N+](=O)[O-])CC3)C(C#N)C2=O)CCC1C1CCC([N+](=O)[O-])CC1</smiles>

4k, $49 \%$

Product 4c was identified as a bis-2-pyridone by spectroscopic analysis, including 2D NMR spectroscopy (Figure 4). The COSY experiment of $\mathbf{4 c}$ confirmed a correlation between the signal of the proton $\left(\mathrm{CH}_{2} ; \mathrm{H}_{\mathrm{A}}\right)$ at $1.36 \mathrm{ppm}$ with that at $1.72 \mathrm{ppm}\left(\mathrm{CH}_{2} ; \mathrm{H}_{\mathrm{B}}\right)$, which exhibits a 
cross peak with the signal at $1.36 \mathrm{ppm}\left(\mathrm{CH}_{2} ; \mathrm{H}_{\mathrm{A}}\right)$ and $3.99 \mathrm{ppm}\left(\mathrm{CH}_{2} ; \mathrm{H}_{\mathrm{C}}\right)$. The signal at 8.14 $\operatorname{ppm}\left(\mathrm{CH} ; \mathrm{H}_{\mathrm{G}}\right)$ exhibits a correlation with that at $6.52 \mathrm{ppm}\left(\mathrm{CH}_{2} ; \mathrm{H}_{\mathrm{G}}\right)$.
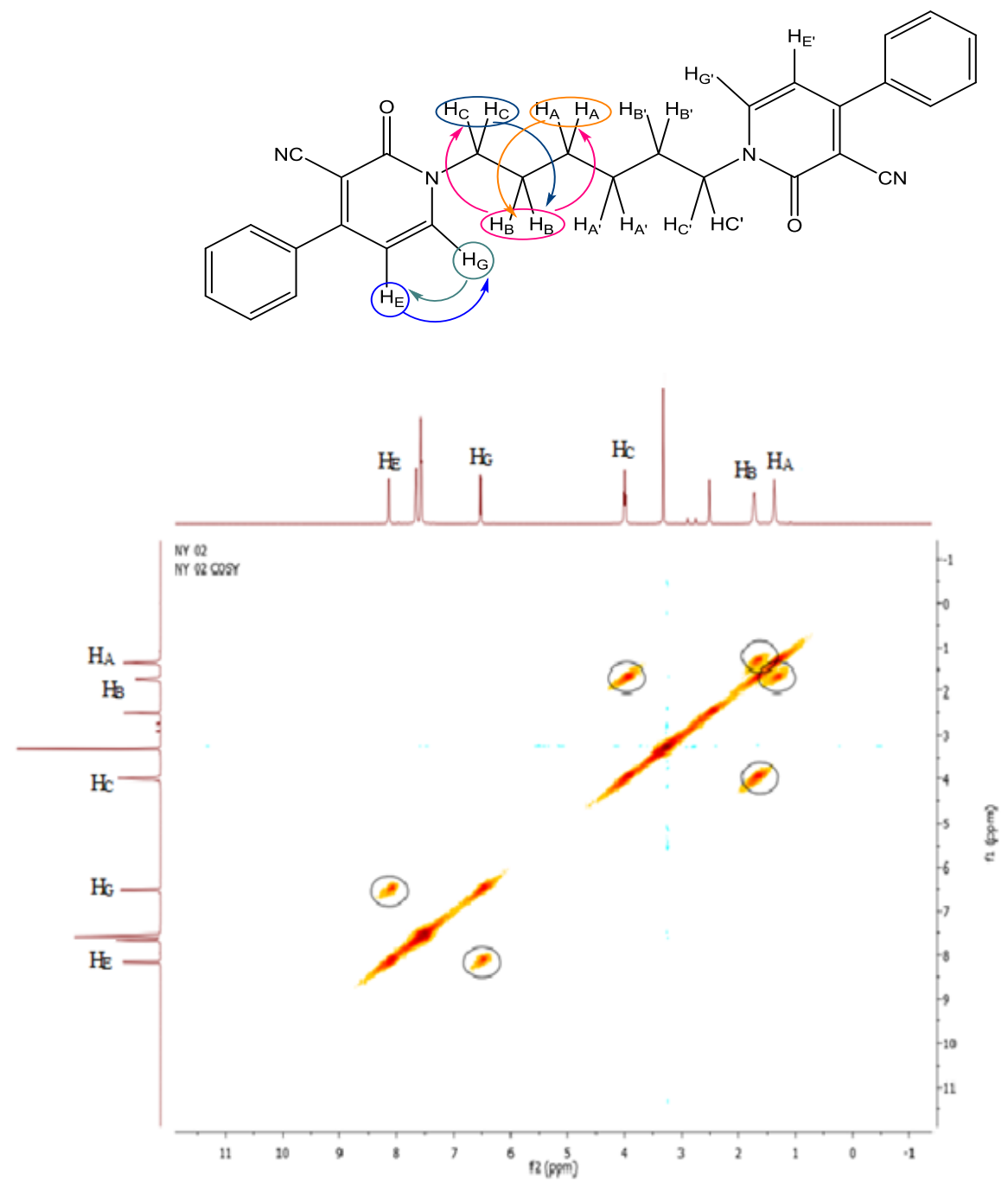

Figure 4.COSY experiment and relative signs of coupling constants of $\mathbf{4 c}$

We have obtained a single crystal of compound $\mathbf{4 c}$ which confirmed its bicyclic structure. Compound $4 \mathbf{c}$ crystallizes in the centrosymmetric monoclinic $P 2_{1} / \mathrm{c}$ space group, with the asymmetric unit being composed of a half of the molecular unit: the remaining portion of the molecule is formed by symmetry through an inversion centre. The close packing in the solid state of individual units is very similar as for the other crystalline compound, mostly promoted by weak hydrogen bonding interactions of the $\mathrm{C}-\mathrm{H} \cdots \mathrm{N}$ and $\mathrm{C}-\mathrm{H} \cdots \mathrm{O}$ types: $\mathrm{d}_{\mathrm{C} \cdots \mathrm{N}}$ distances found in the 3.4395(18)-3.4975(19) $\AA$ range with $<(\mathrm{CHN})$ interaction angles ranging from 151 to $155^{\circ} ; \mathrm{d}_{\mathrm{C} \ldots \mathrm{O}}$ distances were found instead in the 3.2922(17)-3.3809(17) $\AA$ range with the corresponding $<(\mathrm{CHO})$ interaction angles in the range $131-138^{\circ}$. Weak $\pi^{\cdots} \pi$ contacts are present in this case, further promoting a close packing in the solid state with 
inter-centroid distances in range of 3.7007(9)-3.8056(9) $\AA$. The sole presence of weak supramolecular interactions, allied with the high flexibility of the chain between the cyano-2pyridones backbones, leads to the existence of structural disorder for the four central $-\mathrm{CH}_{2}-$ groups of the alkyl chain (Figure 5).

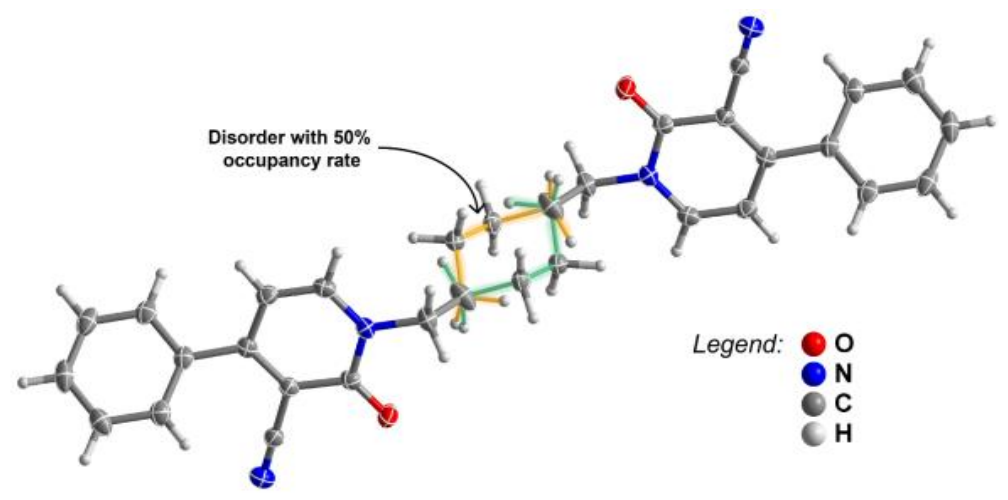

Figure 5. Crystal structure of compound $\mathbf{4 c}$ (The disorder was refined with an occupancy rate of $50 \%$ in each position)

We have study the fluorescence of these compounds in dichloromethane (Figure 6). The absorption and fluorescence emission spectral shapes depend only on the nature of the pyridone and not on the alkyl chain length. All compounds absorb around $350 \mathrm{~nm}$; they emit at $415 \mathrm{~nm}$ while nitro containing compounds $4 \mathrm{i}, 4 \mathrm{j}$ and $4 \mathrm{k}$ emit at $460 \mathrm{~nm}$; however, they are only slightly florescent since their fluorescence quantum yield was measured to be below 0.01 . 


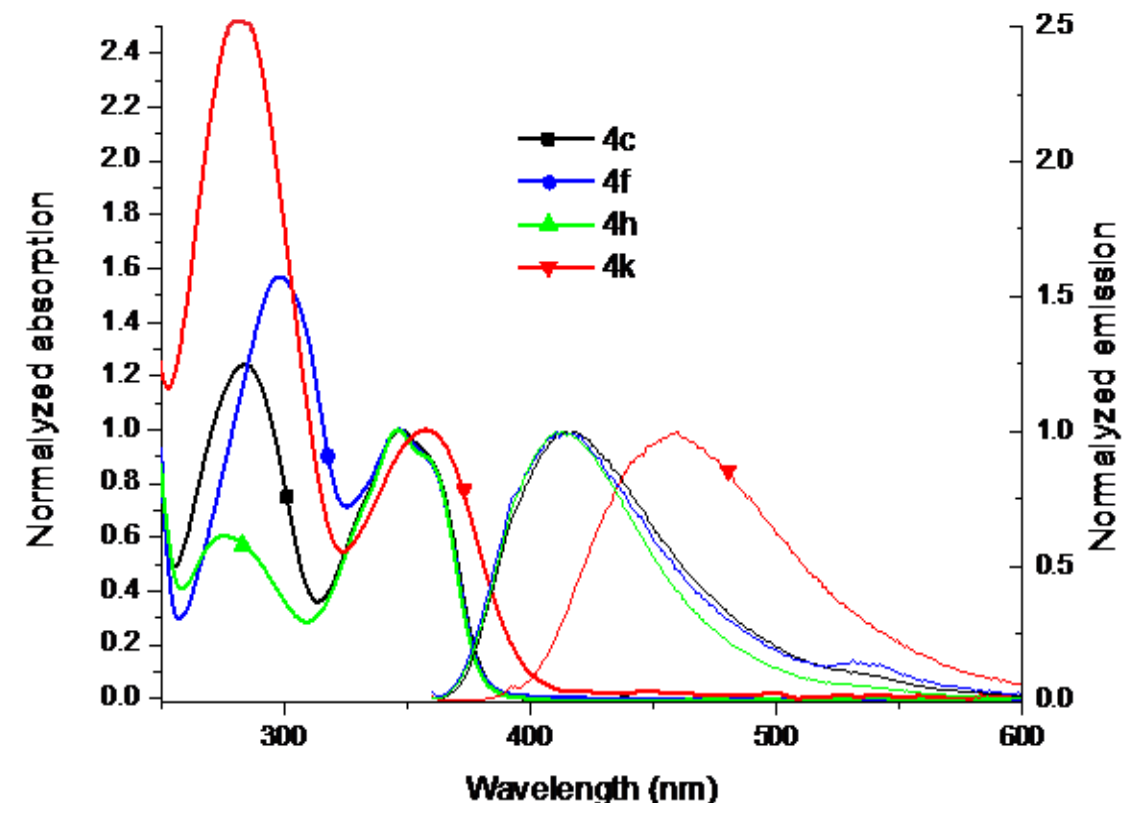

Figure 6. Absorption and fluorescence emission of pyridone dimers in dichloromethane

The mechanism of the described cyclization into bis-2-pyridones is proposed in Scheme 1: starting by a condensation reaction of two equivalents of enaminonitriles I with primary diamines to form intermediate II. The structures of bis-2-pyridones IV were obtained by intramolecular cyclization followed by elimination of two molecules of ethanol.

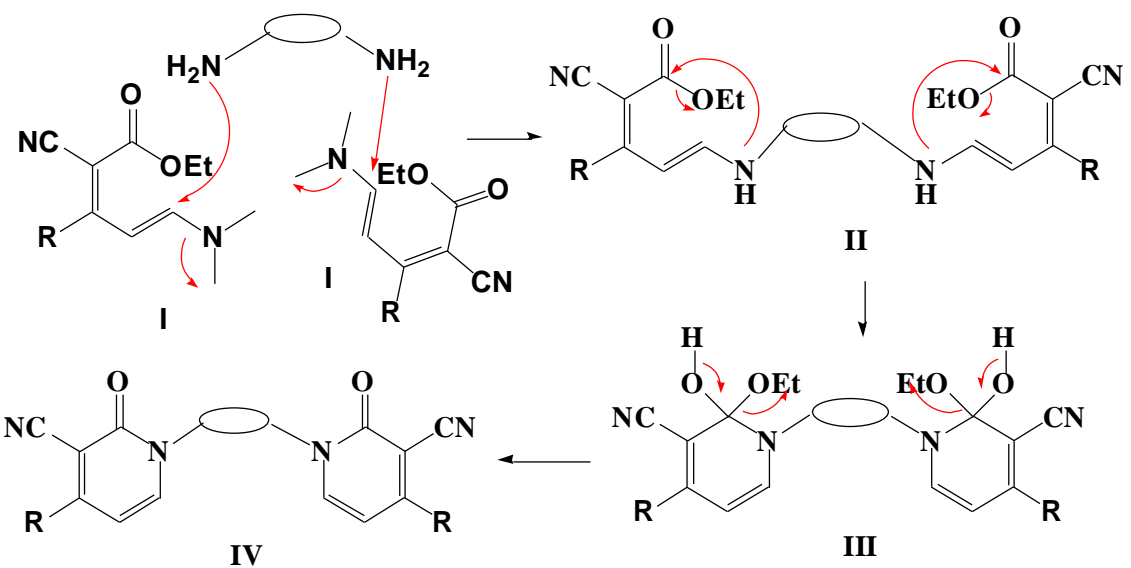

Scheme 1. Proposed mechanism for the synthesis of compounds $4 \mathbf{a}-\mathbf{k}$

\section{Conclusion}

In conclusion, we have developed an efficient procedure to prepare a new series of bis-2pyridones and its various analogues using enaminonitriles as key intermediates and diamines 
in good yields. The fluorescence study demonstrate that the fluorescence depend only on the nature of the pyridone and not on the alkyl chain. The proposed syntheses are simple, with good yields and the scope of this approach could be extended to the preparation of other families of molecules.

\section{Acknowledgements}

The authors wish to thank Directorate General for Scientific Research and Technological Development (DGRSDT) and the University of Tlemcen for the financial support. A part of this work was developed within the scope of the projects CICECO-Aveiro Institute of Materials (FCT UID/CTM/50011/2019) and QOPNA (FCT UID/QUI/00062/2013) through national funds and, where applicable, co-financed by FEDER, within the PT2020 Partnership Agreement. RFM also gratefully acknowledges FCT for a Junior Research Position (CEECIND/00553/2017)

\section{References}

1. D. S. Surry, and S. L. Buchwald, Angewandte Chemie International Edition. 47, 6338 (2008)

2. D. A. Colby, R. G. Bergman, and J. A. Chemical Reviews. 110, 624 (2010)

3. V. V. Krishna Mohan Kandepi, and N. Narender, Catalysis Science \& Technology. 2, 471 (2012)

4. T. Balaban, D. C. Oniciu, and A. R. Katritzky, Chemical Reviews. 104, 2777 (2004)

5. C. Bengtsson, A. E. G. Lindgren, H. Uvell, and F. Almqvist, European Journal of Medicinal Chemistry. 54, 637 (2012)

6. N. C. Desai, N. R. Shihory, and G. M. Kotadiya. Chinese Chemical. Letters. 25, 305 (2014)

7. Z. Lv, C. Sheng, T. Wang, Y. Zhang, J. Liu, J. Feng, H. Sun, H. Zhong, C. Niu, and K. $\mathrm{Li}$, Journal of Medicinal Chemistry. 53, 660 (2010)

8. B. Danieli, G. Lesma, D. Passarella, A. Sacchetti, A. Silvani, and A. Virdis, Organic Letters. 6, 493 (2004)

9. P. Dorigo, R. M. Gaion, P. Belluco, D. Fraccarollo, I. Maragno, G. Bombierij, F. Benetollo, L. Mosti, and F. Orsin, Journal of Medicinal Chemistry. 36, 2475 (1993)

10. M. Asif. Journal of Chemical Reviews 1, 47 (2019)

11. M. Ravinder, B. Mahendar, S. Mattapally, K. V. Hamsini, T. N. Reddy, C. Rohit, K. Srinivas, S. K. Banerjee, and V. J. Rao, Bioorganic \& Medicinal Chemistry Letters 22, 6010 (2012)

12. H. S. P. Rao, and S. Senthilkumar, Current Organic Chemistry. 8, 1521 (2004)

13. Q. Li, L. A. Mitscher, and L. L. Shen.Medicinal Research Reviews 20, 231 (2000)

14. S. Hibi , K. Ueno K, S. Nagato, and al. J Med Chem. 55, 10584 (2012)

15. A. Fassihi, DAbedi, L. Saghaie, R. Sabet, H. Fazeli, G. Bostaki, O. Deilami, and H. Sadinpour. European Journal of Medicinal Chemistry. 44, 2145 (2009)

16. Ž. Mijin, G. S. Ušćumlić, N. V. Valentić, and A. D. Marinković, Hemijskaindustrija 65, 517 (2011)

17. V. Litvinov, S. Krivokolysko, and V. Dyachenko, Chemistry of Heterocyclic Compounds 35, 509 (1999)

18. A-V. Guzzo, D-A. Nelson, Photochemistry and photobiology. 55, 665 (1992) 
19. M. Satoshi, M. Shouta, I. Hiroshi, K. Mitsuo, K. Hirotake , 0. Yutaka , T.Manabu , N. Kiyoyuki, Chemistry Letters.36, 1014( 2007),

20. M. Hagimori, Y.Shigemitsu, R. Murakami, K. Yokota and al. Dyes and Pigments. 124, 196 ( 2016)

21. K-S. Krishnan, C. Bengtsson, J- A-D. Good, S. Mirkhanov, E. Chorell, Lennart B.-A. Johansson, and F. Almqvist . J. Org. Chem. 78, 12207 (2013)

22. L. Comins, H. Hong, and G. Jianhua, Tetrahedron Letters 35, 5331 (1994)

23. S. Hanessian, G. McNaughton-Smith, H. G. Lombart, W. D. Lubell. Tetrahedron 53, 12789 (1997)

24. A. H.Abadi, T. M. Ibrahim, K. M.Aouzid, J. Lehman, H. N. Tinsley, B. D. Gary, G. A. Piazza. Bioorganic \& Medicinal Chemistry 17, 5974 (2009)

25. M. O. Noguez, V. Marcelino, H. Rodríguez, O. Martín, J. O. Martínez, G. A. Arroyo, F. J. Pérez, M. Suárez, and R. Miranda, International Journal of Molecular Sciences. 12, 2641 (2011)

26. M. T. Cocco, C. Congiu, and V. Onnis. European Journal of Medicinal Chemistry 35, $545(2000)$

27. M. T. Cocco, C. Congiu, V. Lilliu, and V. Onnis, European Journal of Medicinal Chemistry.38, 37 (2003)

28. A. J. Demuner, V. M. Moreira Valente, L. C. Almeida Barbosa, A. Rathi, T. J. Donohoe, and A. L. Thompson, Molecules. 14, 4973 (2009)

29. S. M. Sanad, A. H. Elwahy, and I. A. Abdelhamid, Arkivoc vii, 39 (2018)

30. Z. Kibou, N. Cheikh, N. Choukchou-Braham, D. Villemin.J. Mater. Environ. Sci. 7, 3061 (2016)

31. F. Belhadj, Z. Kibou, N. Cheikh, N. Choukchou-Braham, and D. Villemin. Tetrahedron Letters 56, 5999 (2015)

32. Z. Kibou, N. Cheikh,D.Villemin, D.; N. Choukchou-Braham, B. Mostefa-Kara, M. Benabdallah, Scientific Study \& Research, 12 ,121 (2011)

33. Z. Kibou, D. Villemin, J. F. Lohier, N. Cheikh, N. Bar, and N. Choukchou-Braham, Tetrahedron 72, 1653 (2016)

34. F. Nouali, Z. Kibou, Z, B. Boukoussa, N. Choukchou-Braham, A. Bengueddach, D.Villemin,R. Hamacha. Res Chem Intermed 46, 3179 (2020)

35. N. Mehiaoui, Z. Kibou, A. Berrichi, N. Choukchou-Braham. R. Bachir. Res Chem Intermed, (2020) . https://doi.org/10.1007/s11164-020-04261-1

36. T. Kottke, and D. Stalke, Journal of Applied Crystallography 26, 615 (1993)

37. APEX3, Data Collection Software Version 2016. 9-0, Bruker AXS, Delft, The Netherlands, 2005-2016

38. Cryopad, Remote monitoring and control, Version 1.451, Oxford Cryosystems, Oxford, United Kingdom, 2006

39. SAINT+, Data Integration Engine v. $8.37 a^{\circ}, 1997-2015$ Bruker AXS, Madison, Wisconsin, USA

40. L. Krause, R. Herbst-Irmer, G. M. Sheldrick, and D. Stalke,Journal of Applied crystallography 48, 3 (2015)

41. M. Sheldrick, Acta Crystallographica Section A: Foundations and Advances 71, 3 (2015)

42. M. Sheldrick, Acta Crystallographica Section C: Structural Chemistry 71, 3 (2015)

43. C. B. Hübschle, G. M. Sheldrick, and B. Dittrich, Journal of Applied Crystallography 44, 1281 (2011) 
44. K. Brandenburg, "DIAMOND, Version 3.0 a; Crystal Impact GbR: Bonn, Germany, 1997-2014

45. Z. Kibou, N.Cheikh, D.Villemin,N. Choukchou-Braham, B. Mostefa-Kara, M.Benabdallah, Inter. J.Org. Chem, 1, 242 (2011) 